\title{
Experimental Review on Lepton Universality and Lepton Flavour Violation tests in $B$ decays
}

\author{
P. de Simone ${ }^{1, a}$ on behalf of the LHCb Collaboration also including results from the Belle Collaboration \\ ${ }^{1}$ INFN Laboratori Nazionali di Frascati, Via Enrico Fermi, 40, 00044, Frascati, Italy
}

\begin{abstract}
Tests of lepton flavour universality in $B$ decays offer an excellent opportunity to test the Standard Model, and show hints of new physics in analyses performed by the LHCb, Belle and BaBar experiments. Several theoretical models proposed to explain possible violation of lepton flavour universality claim a connection with lepton flavour violation in $B$ decays. These proceedings review the experimental status of the tests of lepton flavour universality and the searches of lepton flavour violation in $B$ decays.
\end{abstract}

\section{Introduction}

Some recent measurements, both in the Flavour Changing Neutral Current (FCNC) $b \rightarrow s \ell^{+} \ell^{-}$and tree level semitauonic $b \rightarrow c$ transitions, hint at a violation of one of the key prediction of the Standard Model (SM): the universality of interactions for leptons of different generations (LFU). The theoretical attempts to explain possible violations of LFU in both processes, introduce new mediators such as $Z^{\prime}$ or lepto-quarks and foresee enhancements of the Lepton Flavour Violation (LFV) phenomena to accessible levels $[1,2]$.

These proceedings focus on the recent tests of LFU in FCNC and in semitauonic $B$ decays and also describes few selected searches of LFV $B$ decays.

The experiments that are mainly involved in these studies are BaBar [3] and Belle [4] at $e^{+} e^{-}$colliders in the USA and in Japan respectively, and the LHCb [5] experiment at the $p p$ collider LHC at CERN in Europe.

The BaBar and Belle experiments have collected data at the $\Upsilon(4 S)$ resonance that decays producing $B \bar{B}$ and $B^{0} \bar{B}^{0}$ pairs. These experiments have the advantage that $B$ mesons are produced in a clean environment with little background and that the well constrained kinematics are very beneficial for reconstructing final states with neutrinos. The BaBar and Belle experiments completed the data taking in 2008 and 2010 and collected $433 \mathrm{fb}^{-1}$ and $711 \mathrm{fb}^{-1}$ of data, respectively.

The $\mathrm{LHCb}$ experiment records data at the LHC, where the $b$ quarks are produced through gluon fusion and thus all $b$-hadron species are created: $B^{+}, B^{0}, B_{s}^{0}, B_{c}^{+}$and $\Lambda_{b}^{0}$. The $B$ hadrons are strongly boosted, which provides an excellent separation between production and decay vertices. However, the large amount of $b$ hadrons created comes at the cost of a lot of background. The LHCb experiment

\footnotetext{
a e-mail: patrizia.desimone@lnf.infn.it
}

recorded $3 \mathrm{fb}^{-1}$ of data in $2011-2012$ at $\sqrt{s}=7-8 \mathrm{TeV}$, and $6 \mathrm{fb}^{-1}$ in $2015-2018$ at $\sqrt{s}=13 \mathrm{TeV}$.

\section{Test of LFU in $B \rightarrow K^{(*)} \ell \ell$ decays}

The FCNC decays, $B \rightarrow K^{(*)} \ell \ell$, are highly suppressed in the SM as they proceed through amplitudes involving electroweak loop diagrams and their branching ratios $(\mathcal{B R})$ are expected to be less than $10^{-6}$. However, the decay ratios defined as

$$
\mathcal{R}_{K^{(*)}}=\frac{\mathcal{B R}\left(B \rightarrow K^{(*)} \mu^{+} \mu^{-}\right)}{\mathcal{B R}\left(B \rightarrow K^{(*)} e^{+} e^{-}\right)}
$$

are predicted to be 1 with $O(1 \%)$ precision in the di-lepton invariant mass squared range $1.1 \leq q^{2} \leq 6 . \mathrm{GeV}^{2} / c^{4}[6]$, allowing for a precise indirect access to New Physics (NP) phenomena.

The LHCb measurements for the LFU ratios $\mathcal{R}_{K^{(*)}}$ as a function of $q^{2}[10,11]$, show a systematic deviation below the SM expectations between 2.2 and $2.6 \sigma$, while the previous measurements by BaBar and Belle [7, 8] have considerably greater uncertainties but are consistent with the SM prediction. This section reports about the update of the ratios $\mathcal{R}_{K}$ and $\mathcal{R}_{K^{*}}$ measured by the $\mathrm{LHCb}$ [12] and Belle [13] collaborations, respectively.

\section{$2.1 \mathcal{R}_{K}$ measurement at $\mathrm{LHCb}$}

The new $\mathcal{R}_{K}$ measurement [12] is based on an optimization of the previous LHCb analysis [10] and on an increased data sample almost doubled, about $5 \mathrm{fb}^{-1}$.

The main challenge of this measurement is to reduce the systematic uncertainties induced by the different behaviour of muons and electrons within the detector. In particular, the former have a clear experimental signature with high reconstruction efficiency, while the latter emits a large amount of bremsstrahlung that results in a degraded $B$ momentum and mass resolution. A bremsstrahlung 

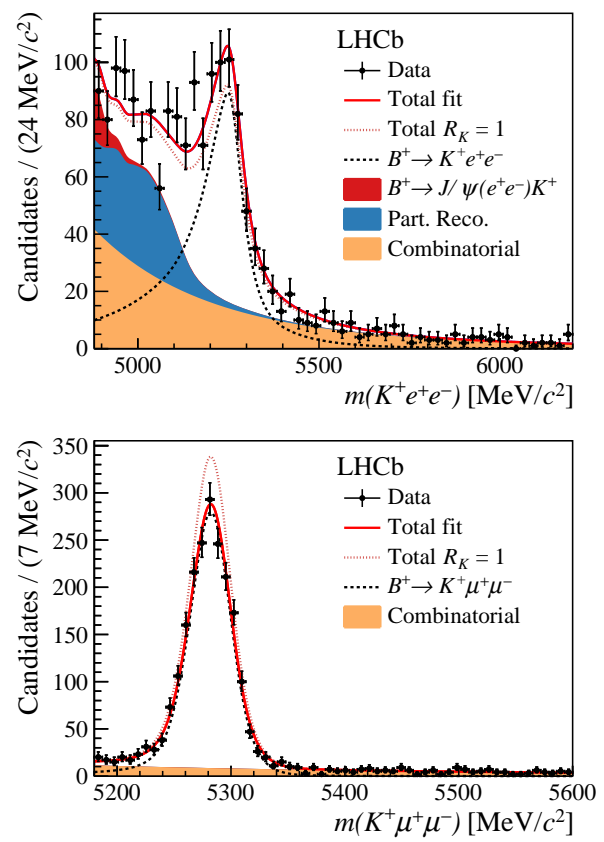

Figure 1: Fits to the $m\left(K^{+} \ell \ell\right)$ invariant mass distributions for (up) electron and (down) muon candidates for nonresonant decays. The red-dotted lines show the distributions that would be expected from the observed number of $B^{+} \rightarrow K^{+} \ell \ell$ decays and $\mathcal{R}_{K}=1$.

recovery algorithm is used to improve the electron momentum reconstruction; the correction is made by adding to the electron the momentum of photons consistent with being emitted from the electron [9]. To minimize the systematic uncertainties, $\mathcal{R}_{K}$ is measured as a double ratio of the rare signal channels with respect to the resonant decays of the $B$ mesons

$\mathcal{R}_{K}=\frac{\mathcal{B R}\left(B^{+} \rightarrow K^{+} \mu^{+} \mu^{-}\right)}{\mathcal{B R}\left(B^{+} \rightarrow K^{+} J / \psi\left(\mu^{+} \mu^{-}\right)\right)} / \frac{\mathcal{B R}\left(B^{+} \rightarrow K^{+} e^{+} e^{-}\right)}{\mathcal{B R}\left(B^{+} \rightarrow K^{+} J / \psi\left(e^{+} e^{-}\right)\right)}$

The candidates for the resonant channels are selected using similar criteria to that of the rare counterpart. An unbinned extended maximum-likelihood fit to the invariant masses of the nonresonant decays $m\left(K^{+} \mu^{+} \mu^{-}\right)$and $m\left(K^{+} e^{+} e^{-}\right)$, is used to count the signal yields. Figure 1 shows the result of the fit.

For the dilepton mass-squared region $1.1<q^{2}<$ $6.0 \mathrm{GeV}^{2} / c^{4}$ the ratio of branching fractions for $B^{+} \rightarrow$ $K^{+} e^{+} e^{-}$and $B^{+} \rightarrow K^{+} \mu^{+} \mu^{-}$is measured to be

$$
\mathcal{R}_{K}=0.846_{-0.054}^{+0.060}(\text { stat })_{-0.016}^{+0.014} \text { (syst) }
$$

This is the most precise measurement of the $\mathcal{R}_{K}$ ratio to date and is consistent with the SM prediction at the level of 2.5 standard deviations. Further reduction in the uncertainty on $\mathcal{R}_{K}$ will be obtained when the remaining data collected by LHCb, which has a statistical power approximately equal to that of the data set used for this measurement, is included in a future analysis.
Table 1: Results for $\mathcal{R}_{K^{*}}, \mathcal{R}_{K^{* 0}}$ and $\mathcal{R}_{K^{*+}}$. The first uncertainties are statistical and the second are systematic.

\begin{tabular}{llll}
\hline$q^{2} \mathrm{GeV}^{2} / c^{4}$ & all modes & $B^{0}$ modes & $B^{+}$modes \\
\hline $0.045-1.1$ & $0.52_{-.26}^{+.36} \pm .07$ & $0.46_{-.27}^{+.55} \pm .07$ & $0.62_{-.36}^{+.60} \pm .10$ \\
$1.1-6$ & $0.96_{-.29}^{+.45} \pm .11$ & $1.06_{-.38}^{+.63} \pm .13$ & $0.72_{-.44}^{+.99} \pm .18$ \\
$0.1-8$ & $0.90_{-.21}^{+.27} \pm .10$ & $0.86_{-.24}^{+.33} \pm .08$ & $0.96_{-.35}^{+.56} \pm .14$ \\
$15-19$ & $1.18_{-.32}^{+.52} \pm .10$ & $1.12_{-.36}^{+.61} \pm .10$ & $1.40_{-.68}^{+1.99} \pm .11$ \\
0.045 & $0.94_{-.14}^{+.17} \pm .08$ & $1.12_{-.21}^{+.27} \pm .09$ & $0.70_{-.19}^{+.24} \pm .07$ \\
\hline
\end{tabular}

\section{$2.2 \mathcal{R}_{K^{*}}$ measurement at Belle}

Recently the Belle collaboration presented a new measurement of the ratio $\mathcal{R}_{K^{*}}$ for both charged and neutral $B$ mesons, using the full data sample, containing $772 \times 10^{6} \mathrm{~B} \bar{B}$ events [13], recorded at the $\Upsilon(4 S)$ resonance at the KEKB asymmetric-energy $e^{+} e^{-}$collider. Neutral and charged $B$ mesons are reconstructed in final states with a $K^{*}$ and a couple of muons or electrons. The $K^{*}$ candidates are formed from $K^{+} \pi^{+}, K^{+} \pi^{0}$ and $K_{\mathrm{S}}^{0} \pi^{+}$combinations with an invariant mass in the range $0.6 \mathrm{GeV} / c^{2}<M_{K \pi}<1.4 \mathrm{GeV} / c^{2}$. The yields of the $B \rightarrow K^{*} \ell \ell$ candidates, are extracted in various regions of $q^{2}$, with an unbinned extended maximum likelihood fit to the distribution of the beam energy constrained mass, defined as

$$
M_{b c}=\sqrt{E_{\text {beam }}^{2}-p_{B}^{2}}
$$

where $E_{\text {beam }}$ is the beam energy and $p_{B}$ is the momentum of the reconstructed $B$ meson candidate; these quantities are evaluated in the $e^{+} e^{-}$center-of-mass (CM) frame.

Using the fitted signal yields, the lepton flavour universality ratio $\mathcal{R}_{K^{*}}$ is evaluated for all signal channels combined, as well as separate ratios for the $B^{0}$ and $B^{+}$decays, $\mathcal{R}_{K^{* 0}}$ and $\mathcal{R}_{K^{*+}}$. The results are shown in table 1 . These measurements, all consistent with the SM expectations, will became competitive with the larger data sample that will be collected at the Belle II experiment [14, 15], expected to be about 50 times larger than the Belle data sample.

\section{Test of LFU in $b \rightarrow c \tau \nu_{\tau}$ transitions}

This section reviews the tests of LFU violations in chargedcurrent $B$ decays which are of the form $b \rightarrow c \tau v_{\tau}$, commonly done through the measurements of the ratios

$$
\mathcal{R}\left(H_{c}\right)=\frac{\mathcal{B R}\left(H_{b} \rightarrow H_{c} \tau v_{\tau}\right)}{\mathcal{B R}\left(H_{b} \rightarrow H_{c} \ell v_{\ell}\right)}
$$

where $\mathcal{R}\left(H_{b}\right)$ and $\mathcal{R}\left(H_{c}\right)$ are $b$ and $c$ hadrons respectively, and $\ell$ is either an electron or a muon. The semitauonic decay is called the signal channel, and the other decay is the normalisation channel. Up until the start of 2019, the combination of all the measurements for the ratios $\mathcal{R}(D)$ and $\mathcal{R}\left(D^{*}\right)$ [17-22] was showing a tension of about $4 \sigma$ with the SM prediction. 


\subsection{Muonic $\mathcal{R}\left(D^{*}\right)$ and $\mathcal{R}(J / \psi)$ measurements at $\mathrm{LHCb}$}

The $\mathcal{R}\left(D^{*}\right)$ analysis [19] measures the ratio

$$
\mathcal{R}\left(D^{*}\right)=\frac{\mathcal{B R}\left(\overline{B^{0}} \rightarrow D^{*+} \tau^{-} \overline{v_{\tau}}\right)}{\mathcal{B R}\left(\bar{B}^{0} \rightarrow D^{*+} \mu^{-} \overline{v_{\mu}}\right)}
$$

The $\tau$ lepton in its leptonic decay, $\tau^{-} \rightarrow \mu^{-} \overline{v_{\mu}} v_{\tau}$, is selected, which means that the signal and the normalisation channels both have the same visible final state: 3 charged tracks from the decay chain $D^{*} \rightarrow D^{0}(\rightarrow K \pi) \pi$, and 1 track identified as a muon. This implies the cancellation of many systematic uncertainties in the ratio. The yields of the signal, the normalisation components and the residual background are statistically disentangled with a multidimensional template fit based on three kinematic variables evaluated in the $B$ meson rest frame: the missing mass squared, $m_{m i s s}^{2}$, the muon energy, $E_{\mu}^{*}$, and the square of the fourmomenta transfer to the lepton system $\left(\ell v_{\ell}\right), q^{2}$. Thanks to the excellent performance of the $\mathrm{LHCb}$ vertex detector the flight direction of the $B$ mesons is precisely determined, from which the transverse component of the missing momentum is deduced. Then, the momentum component along the beam direction of the $B$ meson is approximated with the boost of the visible final state: $\left(\gamma \beta_{z}\right)_{B} \simeq\left(\gamma \beta_{z}\right)_{D^{*} \mu}$.

The analysis is performed using the first $3 \mathrm{fb}^{-1}$ collected at $\mathrm{LHCb}$. The result of the fit, in the highest $q^{2}$ bin, is shown in figure 2. After correcting for the reconstruction and selection efficiencies, the signal and normalisation yields give a value of

$$
\mathcal{R}\left(D^{*}\right)=0.336 \pm 0.027(\text { stat }) \pm 0.030 \text { (syst) } .
$$

The largest contribution to the systematic uncertainty is due to the limited size of the simulation samples used to model the template shapes. The obtained value of $\mathcal{R}\left(D^{*}\right)$ is $1.9 \sigma$ above the SM expectations.

The studies of semitauonic decays of other $b$-hadron species can provide an additional handle for investigating the sources of theoretical and experimental uncertainties; the LHCb collaboration started these studies with the measurement of the ratio [24]

$$
\mathcal{R}(J / \psi)=\frac{\mathcal{B R}\left(B_{c}^{+} \rightarrow J / \psi \tau^{+} v_{\tau}\right)}{\mathcal{B R}\left(B_{c}^{+} \rightarrow J / \psi \mu^{+} v_{\mu}\right)}
$$

The $\tau$ lepton is reconstructed in the muonic decay mode and the signal and normalisation channels are identified through their identical visible final state, two muon candidates from the $J / \psi$ decay and one from the semileptonic decay of the $B_{c}^{+}$. As in the $\mathcal{R}\left(D^{*}\right)$ analysis, signal and normalisation events are distinguished with a three dimensional fit based on kinematic variables evaluated in the $B_{c}^{+}$reference system, and on the $B_{c}^{+}$lifetime that is about 3 times longer with respect to that of the lighter $b$-mesons. The analysis yields a value of

$$
\mathcal{R}(J / \psi)=0.71 \pm 0.17(\text { stat }) \pm 0.18(\text { syst }),
$$

where one of the largest systematic contribution comes from the limited knowledge on the form factors; these
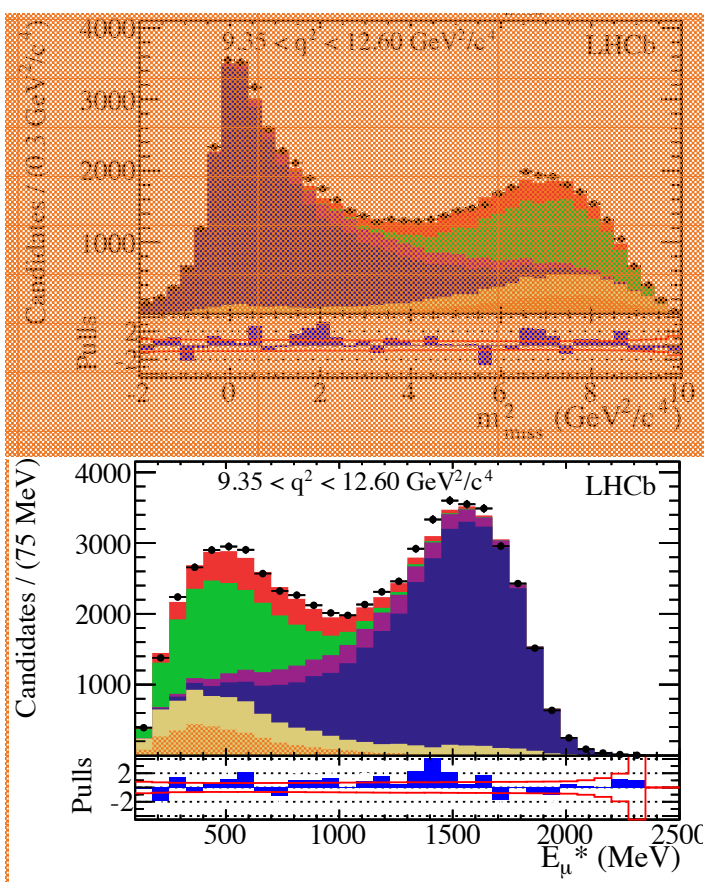

Figure 2: Distributions of $m_{m i s s}^{2}$ (up) and $E_{\mu}^{*}$ (down) in the highest $q^{2}$ bin of the selected data sample, overlaid with the projections of the fit model. The signal component is drawn in red, while the normalisation in blue. Background components of the selected samples are the doubly charmed decays, $H_{b} \rightarrow H_{c} H_{c}(\rightarrow \ell \tau X)$, in green, the $B \rightarrow D^{* *} \ell v$ decays in violet, combinatorial and misidentified muons in light and dark yellow, respectively.

are currently evaluated on a subsample enriched in the normalisation mode, but can be significantly improved with new lattice calculations. The obtained value of $\mathcal{R}(J / \psi)$ is 2. $\sigma$ above the SM expectations.

\subsection{Hadronic $\mathcal{R}\left(D^{*}\right)$ measurement at $\mathbf{L H C b}$}

The $\mathcal{R}\left(D^{*}\right)$ measurement has been repeated reconstructing the $\tau$ decays in three charged pions, $\tau^{ \pm} \rightarrow \pi^{ \pm} \pi^{+} \pi^{-}\left(\pi^{0}\right) \nu_{\tau}$, $[22,23]$. The $D^{*}$ meson is reconstructed through the same decay chain as in the muonic $\mathcal{R}\left(D^{*}\right)$ analysis, it follows that the visible final state of the semitauonic signal consists of 6 charged tracks. To minimize the experimental systematic uncertainties the signal counting is normalised to the yield of the $\bar{B}^{0} \rightarrow D^{*+} \pi^{-} \pi^{+} \pi^{-}$decay, and the measured quantity is the ratio

$$
\mathcal{K}\left(D^{*}\right)=\frac{\mathcal{B R}\left(\overline{B^{0}} \rightarrow D^{*+} \tau^{-} \overline{v_{\tau}}\right)}{\mathcal{B R}\left(\bar{B}^{0} \rightarrow D^{*+} \pi^{-} \pi^{+} \pi^{-}\right)} .
$$

To convert this value to $\mathcal{R}\left(D^{*}\right), \mathcal{K}\left(D^{*}\right)$ is multiplied by the ratio of the branching ratios of the $\bar{B}^{0} \rightarrow D^{*+} \pi^{-} \pi^{+} \pi^{-}$and $\bar{B}^{0} \rightarrow D^{*+} \mu^{-} \bar{v}_{\mu}$ decays, which are taken as external inputs from HFLAV average:

$$
\mathcal{R}\left(D^{*}\right)=\mathcal{K}\left(D^{*}\right) \times\left(\frac{\mathcal{B} \mathcal{R}\left(\bar{B}^{0} \rightarrow D^{*+} \pi^{-} \pi^{+} \pi^{-}\right)}{\mathcal{B R}\left(\bar{B}^{0} \rightarrow D^{*+} \mu^{-} \bar{v}_{\mu}\right)}\right) .
$$

This analysis benefits from the 3-tracks topology of the $\tau$ decay, that enables for a precise reconstruction of the $\tau$ 
vertex, well detached from the $B$ meson decay. The yields of semitauonic signal and normalisation $B \rightarrow D^{*} 3 \pi$ decays, are determined with a three dimensional binned maximumlikelihood fit to the distributions of $q^{2}$, the decay time of the three pions, and the output of a boosted decision tree used to suppress backgrounds coming mainly from doublycharmed decays, $X_{b} \rightarrow D^{*} D_{(s)} X$, where $X_{b}=B^{+}, B^{0}, B_{s}^{0}$. The measured value of $\mathcal{K}\left(D^{*}\right)$ is

$$
\mathcal{K}\left(D^{*}\right)=1.93 \pm 0.12(\text { stat }) \pm 0.17 \text { (syst) }
$$

Using the last updated value for the semimuonic $B^{0}$ decay from HFLAV

$$
\mathcal{B R}\left(\overline{B^{0}} \rightarrow D^{*+} \mu^{-} \overline{v_{\mu}}\right)=(5.08 \pm 0.02 \pm 0.12) \%,
$$

the $\mathrm{LHCb}$ measured value of the hadronic $\mathcal{R}\left(D^{*}\right)$ is

$$
\mathcal{R}\left(D^{*}\right)=0.280 \pm 0.018 \text { (stat) } \pm 0.029 \text { (syst) },
$$

in agreement with the SM within $1 \sigma$.

\subsection{Last simultaneous measurement of $\mathcal{R}(D)$ and $\mathcal{R}\left(D^{*}\right)$ at Belle}

The latest LFU test of the Belle collaboration [25] is a simultaneous measurement of $\mathcal{R}(D)$ and $\mathcal{R}\left(D^{*}\right)$. It uses the full data sample recorded by the Belle detector. The $B \bar{B}$ pairs produced at the $\Upsilon(4 S)$ resonance can be tagged reconstructing an hadronic or a semileptonic decay of one of the two $B$ mesons, referred to as $B_{\text {tag }}$. This last analysis uses a semileptonic tag algorithm; these algorithms exploit the larger branching fractions available, however the presence of the neutrino leads to a weaker constrain on the $B_{\text {signal }}$. Since the previous $\mathcal{R}\left(D^{*}\right)$ analysis with a semileptonic tag [20], the tagging algorithm has been improved with a new multivariate analysis to improve the efficiency and purity of the semileptonic $B_{\text {tag }}$ sample.

Neutral and charged $B$ mesons are reconstructed in four data samples, $D^{+} \ell^{-}, D^{0} \ell^{-}, D^{*+} \ell^{-}$and $D^{* 0} \ell^{-}$decays, with $\ell=e, \mu$, which increases the signal yields compared to the previous analysis [20] where only $B^{0}$ decays were considered. The $D^{0}$ and $D^{+}$mesons are reconstructed in various final states with kaons and pions, adding up 30\% and $22 \%$ of the total $D^{0}$ and $D^{+}$branching fraction, respectively. In each event, the two $B$ mesons are required to have opposite flavour to reduce the combinatorial background.

To distinguish signal and normalisation events from the background is used the sum of the energy of neutral calorimetric clusters that are not associated to reconstructed particles, denoted as $E_{E C L}$. Signal and normalisation events peak near zero in $E_{E C L}$, while background events have a reasonably flat distribution. For each of the four samples, the yields of signal and normalisation events are extracted with a two-dimensional template fit to the distributions of $E_{E C L}$ and a BDT classifier, named CLASS. The input variables to the BDT are the measured visible energy, $E_{v i s}$, the approximate missing mass squared, $m_{\text {miss }}^{2}$, and the angle between the $B$ meson and the $D^{(*)} \ell$ combination in the $\Upsilon(4 S)$ rest frame, $\cos \theta_{B, D^{(*)} \ell}$.

As an example, figure 3 shows the fit projections of the $D^{* 0} \ell^{-}$sample. The blue signal sample is hardly visible
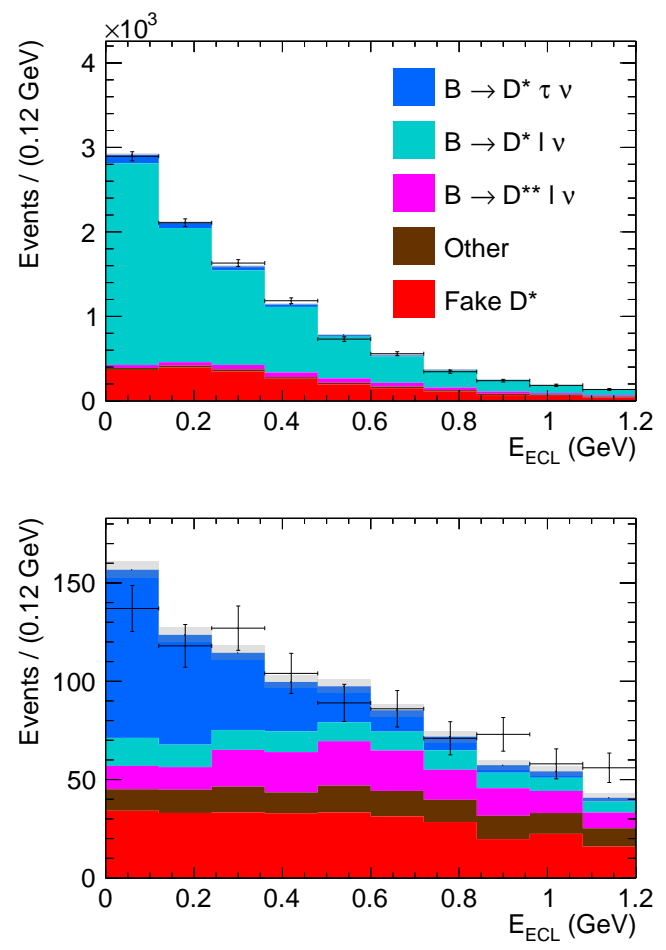

Figure 3: Fit projections of the $D^{* 0} \ell^{-}$sample as a function of $E_{\mathrm{ECL}}$. The upper plot shows the full classifier region, while the lower plot shows the signal region, defined by the cut class $>0.9$.

in the upper plot showing the full classifier region. To illustrate the region associated with the signal, the fit result for the region with class $>0.9$ is shown in the lower plot of figure 3 . Here, the signal is much more visible, and the contribution of the normalisation channel is reduced. Finally, $\mathcal{R}\left(D^{(*)}\right)$ can be calculated using the expression

$$
\mathcal{R}\left(D^{(*)}\right)=\frac{1}{2 \mathcal{B R}\left(\tau^{-} \rightarrow \ell^{-} \bar{v}_{\ell} \nu_{\tau}\right)} \cdot \frac{\varepsilon_{\mathrm{norm}}}{\varepsilon_{\mathrm{sig}}} \cdot \frac{N_{\mathrm{sig}}}{N_{\mathrm{norm}}},
$$

where $\varepsilon_{\text {sig(norm) }}$ and $N_{\text {sig(norm) }}$ are the detection efficiency and fitted yields of the signal and normalisation modes, respectively. $\mathcal{B R}\left(\tau^{-} \rightarrow \ell^{-} \bar{v}_{\ell} v_{\tau}\right)$ is the world average for $\ell=e, \mu$. The efficiencies are taken from simulation samples, which are corrected to resemble the data more closely by applying correction factors; corrections are evaluated using control samples of $e^{+} e^{-} \rightarrow e^{+} e^{-} \ell^{+} \ell^{-}$and $J / \psi \rightarrow \ell^{+} \ell^{-}$ decays.

The analysis measures

$$
\begin{aligned}
\mathcal{R}(D) & =0.307 \pm 0.037 \text { (stat) } \pm 0.016 \text { (syst) } \\
\mathcal{R}\left(D^{*}\right) & =0.283 \pm 0.018 \text { (stat) } \pm 0.014 \text { (syst), }
\end{aligned}
$$

where the correlation between the statistical uncertainties and between the systematic uncertainties is -0.53 and -0.52 , respectively. These are the most precise measurements of $\mathcal{R}(D)$ and $\mathcal{R}\left(D^{*}\right)$ to date and they are in agreement with the SM within $0.2 \sigma$ and $1.1 \sigma$, respectively. The combined result agrees with the SM prediction within $1.2 \sigma$. The largest contributions to the systematic uncertainties come from the limited size of the simulation sample and from the knowledge on the reconstruction efficiency. 


\subsection{New HFLAV average}

The HFLAV group produced new averages for all measurements of $\mathcal{R}(D)$ and $\mathcal{R}\left(D^{*}\right)$ including the latest result from Belle [25]. The current averages are

$$
\begin{aligned}
\mathcal{R}(D) & =0.349 \pm 0.027 \text { (stat) } \pm 0.015 \text { (syst) } \\
\mathcal{R}\left(D^{*}\right) & =0.298 \pm 0.011 \text { (stat) } \pm 0.007 \text { (syst) }
\end{aligned}
$$

The combination of all measurements of $\mathcal{R}(D)$ and $\mathcal{R}\left(D^{*}\right)$,

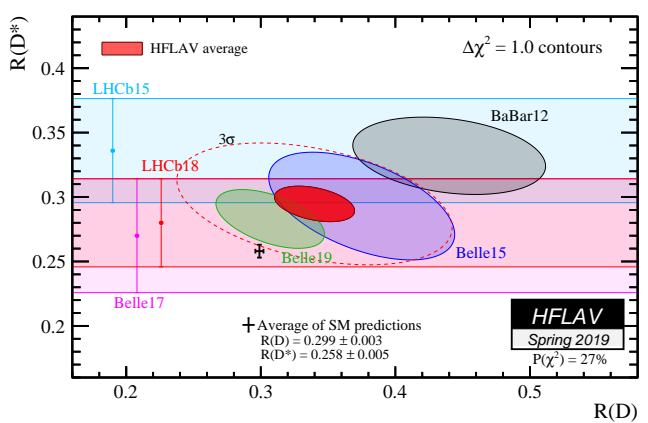

Figure 4: HFLAV average of all measurements of $\mathcal{R}(D)$ and $\mathcal{R}\left(D^{*}\right)$, updated with the latest result of the Belle collaboration presented in these proceedings. The red ellipse shows the combined average and the data point is the SM prediction, showing a discrepancy of 3.1 $\sigma$. This plot is retrieved from the HFLAV website [26].

which is shown in figure 4 , yields a $3.1 \sigma$ discrepancy with the SM [26].

\section{Searches of LFV decays}

The observation of neutrino oscillations has provided the first evidence for Lepton Flavour Violating in neutral leptons. By contrast, LFV processes in the charged sector are extremely suppressed. LFV in heavy hadrons decays may occur within the SM via one-loop diagrams with neutrino oscillations with expected branching fractions of order $10^{-54}$ [27], well beyond current and future experimental sensitivities. However, many theoretical models proposed to explain the possible experimental tensions observed in $B$ meson decays, discussed in the previous sections 2 and 3 , naturally allow for branching fractions that are within the current sensitivity.

This section reports about few recent searches of LFV $B$ meson decays performed by the LHCb and Belle collaborations.

\subsection{Search of $B_{d, s} \rightarrow e \mu$ at LHCb}

NP models with a new gauge $Z^{\prime}$ boson [28] or leptoquarks [29, 30] predict significantly enhanced branching fractions of the $B_{s}^{0} \rightarrow e^{ \pm} \mu^{\mp}$ and $B^{0} \rightarrow e^{ \pm} \mu^{\mp}$ decays, up to $10^{-11}$. The most stringent published limits are currently $\mathcal{B R}\left(B_{s}^{0} \rightarrow e^{ \pm} \mu^{\mp}\right)<1.4 \times 10^{-8}$ and $\mathcal{B R}\left(B^{0} \rightarrow e^{ \pm} \mu^{\mp}\right)<$ $3.7 \times 10^{-9}$ at $95 \%$ confidence level (CL) from the $\mathrm{LHCb}$ collaboration using data corresponding to $1 \mathrm{fb}^{-1}$ of integrated luminosity [31].
Recently a new analysis [32] has been performed on a larger data sample, corresponding to an integrated luminosity of $3 \mathrm{fb}^{-1}$ of $p p$ collisions collected at centre-of-mass energies of 7 and $8 \mathrm{TeV}$ by the LHCb experiment in 2011 and 2012. In addition to a larger data sample, this analysis benefits from an improved selection and in particular a better performing multivariate classifier for signal and background separation. Two normalisation channels are used: the $B^{0} \rightarrow K^{+} \pi^{-}$decay which has a similar topology to that of the signal, and the $B^{+} \rightarrow J / \psi K^{+}$decay, with $J / \psi \rightarrow \mu^{-} \mu^{+}$, which has an abundant yield and a similar trigger selection.

The $B_{d, s} \rightarrow e \mu$ candidates in the events passing the trigger selection are reconstructed by combining pairs of tracks identified as an electrons and a muons, and producing good quality secondary vertices well separated from any Primary Vertex (PV). The measured momentum of electron candidates is corrected for the loss of momentum due to bremsstrahlung [9] (see section 2.1). Since bremsstrahlung can affect the evaluation of selection efficiencies and mass shapes, the selected sample is split into two categories: $B_{d, s} \rightarrow e \mu$ candidates in which no photon is associated with the electron and candidates for which one or more photons are recovered.

Simulated samples are used to evaluate geometrical, reconstruction and selection efficiencies for both signal and backgrounds, to train multivariate classifiers and to determine the shapes of invariant mass distributions, $m_{e \mu}$, of both signal and backgrounds. Trigger and particle identification efficiencies are evaluated directly on high-purity data control samples. The main background components that affect the selection are:

- combinatorial background rejected by means of a Boosted Decision Tree classifier based on the topological properties of the signal events;

- $B \rightarrow h^{+} h^{-}$decays where both hadrons are misidentified as an electron-muon pair;

- semileptonic $B^{0}$ and $\Lambda_{b}^{0}$ decays with a pion misidentified as an electron.

The expected number of events from each possible background source that pass the signal selection is evaluated using the simulation.

The $B^{0} \rightarrow e^{ \pm} \mu^{\mp}$ and $B_{s}^{0} \rightarrow e^{ \pm} \mu^{\mp}$ yields are obtained from a single unbinned extended maximum likelihood fit performed simultaneously to the $m_{e \mu}$ distributions in the two bremsstrahlung categories, which are further divided into seven bins of the BDT classifier. No excesses are observed for these two modes and upper limits on the branching fractions are set to

$$
\begin{aligned}
& B R\left(B_{s}^{0} \rightarrow e^{ \pm} \mu^{\mp}\right)<6.3(5.4) \times 10^{-9} \text { at } 95 \%(90 \%) \mathrm{CL}, \\
& B R\left(B^{0} \rightarrow e^{ \pm} \mu^{\mp}\right)<1.3(1.0) \times 10^{-9} \text { at } 95 \%(90 \%) \mathrm{CL},
\end{aligned}
$$

where only a contribution from the heavy eigenstate is assumed for the $B_{s}^{0}$ meson. If the $B_{s}^{0}$ amplitude is completely dominated by the light eigenstate, the upper limit on the branching fraction becomes

$B R\left(B_{s}^{0} \rightarrow e^{ \pm} \mu^{\mp}\right)<7.2(6.0) \times 10^{-9}$ at $95 \%(90 \%) \mathrm{CL}$. 


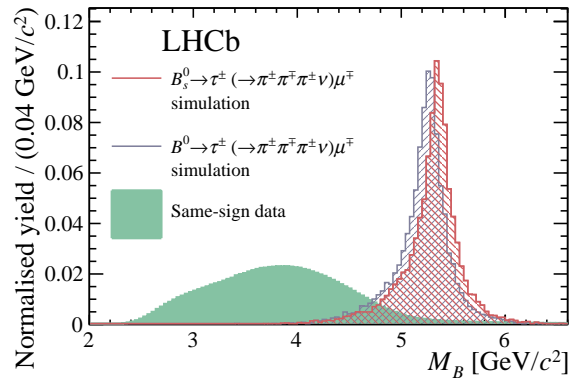

Figure 5: Normalized distributions of the reconstructed invariant mass for $B_{s}^{0}$ and $B^{0}$ in simulated event samples and for same-sign candidates in data.

These results represent the best upper limits to date and are a factor of 2 to 3 better than the previous results from $\mathrm{LHCb}[31]$.

\subsection{Search of $B_{d, s} \rightarrow \tau \mu$ at $\mathbf{L H C b}$}

Recently $\mathrm{LHCb}$ presented the first search for the decay $B_{s}^{0} \rightarrow \tau^{ \pm} \mu^{\mp}$, along with the most stringent limit on the process $B^{0} \rightarrow \tau^{ \pm} \mu^{\mp}$ [33]. An upper limit $\mathcal{B R}\left(B^{0} \rightarrow \tau^{ \pm} \mu^{\mp}\right)<$ $2.2 \times 10^{-5}$ at $90 \%$ CL was obtained by the BaBar collaboration [34]. As in the previous analysis, this search is performed on data corresponding to an integrated luminosity of $3 \mathrm{fb}^{-1}$ of proton-proton collisions, recorded during the years 2011 and 2012 .

The $\tau$ leptons are reconstructed through the decay $\tau^{-} \rightarrow \pi^{+} \pi^{-} \pi^{-} \nu$, which mainly proceeds via the production of two intermediate resonances, $a_{1}(1260)^{-} \rightarrow \pi^{+} \pi^{-} \pi^{-}$ and $\rho(770)^{0} \rightarrow \pi^{+} \pi^{-}$[35], which help in the signal selection. The signal yield is determined by performing an unbinned maximum-likelihood fit to the reconstructed $B$ meson invariant-mass distribution and is converted into a branching fraction using the decay $B^{0} \rightarrow D^{-}\left(\rightarrow K^{+} \pi^{-} \pi^{-}\right) \pi^{+}$ as a normalisation channel. Both signal and normalisation candidates are reconstructed using tracks forming good quality vertices well separated from any PV, with particle identification information corresponding to their assumed particle hypotheses. While the neutrino from the $\tau$ decay escapes detection, its momentum vector can be constrained from the measured positions of the primary and $\tau$ decay vertices, the momenta of the muon and the three pions, and the trajectory of the muon; the invariant mass of the $B_{d, s} \rightarrow \tau \mu$ candidate, $M_{B}$, can be determined analytically up to a twofold ambiguity. The distributions of $M_{B}$ for candidates satisfying the initial selection in the simulated signal samples and in a same-sign data control sample are shown in figure 5. To reduce the data to a manageable level and focus on the rejection of the most difficult backgrounds, the low-mass region with $M_{B}<4 \mathrm{GeV} / c^{2}$ is discarded.

More than $90 \%$ of the background is due to decays with additional reconstructed particles and is rejected using isolation criteria. Two background components survive:

- the partially reconstructed $B$ meson decays;
Table 2: Expected and observed 90\% and 95\% CL limits on the $B_{d, s} \rightarrow \tau \mu$ branching fraction.

\begin{tabular}{c|c|c|c}
\hline Mode & Limit & $90 \%$ CL & $95 \%$ CL \\
\hline \hline$B_{s}^{0} \rightarrow \tau^{ \pm} \mu^{\mp}$ & Observed & $3.4 \times 10^{-5}$ & $4.2 \times 10^{-5}$ \\
& Expected & $3.9 \times 10^{-5}$ & $4.7 \times 10^{-5}$ \\
\hline$B^{0} \rightarrow \tau^{ \pm} \mu^{\mp}$ & Observed & $1.2 \times 10^{-5}$ & $1.4 \times 10^{-5}$ \\
& Expected & $1.6 \times 10^{-5}$ & $1.9 \times 10^{-5}$ \\
\hline
\end{tabular}

- the residual combinatorial background rejected with a Multivariate Analysis techniques.

A final BDT has been trained on a simulated signal sample to split the selected candidates into 4 samples with different signal to background ratios.

No excesses are observed and limits on the $B_{s}^{0} \rightarrow \tau^{ \pm} \mu^{\mp}$ and $B^{0} \rightarrow \tau^{ \pm} \mu^{\mp}$ branching fractions at $90 \%$ and $95 \% \mathrm{CL}$ are given in Table 2 . These results constitute a factor $\sim 2$ improvement with respect to the $\mathrm{BaBar}$ result for the $B^{0}$ mode [34] and the first measurement for the $B_{s}^{0}$ mode.

\subsection{Search of $B^{0} \rightarrow K^{* 0} \mu^{ \pm} e^{\mp}$ at Belle}

Another way to search for LFV is via the decays $B^{0} \rightarrow$ $K^{* 0} \mu^{ \pm} e^{\mp}$, which have large available phase space and also avoid the helicity suppression that a two-body decay such as $B^{0} \rightarrow \mu^{ \pm} e^{\mp}$ might be subjected to. The most stringent upper limits for $B^{0} \rightarrow K^{* 0} \mu^{ \pm} e^{\mp}$ were set by the BaBar experiment based on a data sample of $229 \times 10^{6} B \bar{B}$ events [37]. Recently the Belle collaboration published the search for $B^{0} \rightarrow K^{* 0} \mu^{ \pm} e^{\mp}$ using their full data sample [38].

$B^{0} \rightarrow K^{* 0} \mu^{ \pm} e^{\mp}$ decays are reconstructed by selecting charged particles that originate from a region near the $e^{+} e^{-}$ interaction point. Kaon and pion candidates are combined to form $K^{* 0}$ candidates, and the $B$ candidates are subsequently reconstructed by combining $K^{* 0}$ with $\mu^{ \pm}$, and $e^{\mp}$ candidates. To discriminate signal decays from background, two kinematic variables are defined: the beam-energyconstrained mass, $M_{b c}$, already defined in section 2.2 and the energy difference $\Delta E=E_{B}-E_{\text {beam }}$, where $E_{\text {beam }}$ is the beam energy and $E_{B}$ is the energy of the $B$ candidate. These quantities are evaluated in the $e^{+} e^{-} \mathrm{CM}$ frame.

The most significant background comes from $e^{+} e^{-} \rightarrow$ $q \bar{q}(q=u, d, s, c)$ continuum events. As lighter quarks are produced with large initial momentum, these events tend to consist of two back-to-back jets of pions and kaons. In contrast, $e^{+} e^{-} \rightarrow b \bar{b}$ events result in $B \bar{B}$ pairs produced almost at rest in the $\mathrm{CM}$ frame; this results in more spherically distributed daughter particles. A discriminating variable, based on event topology and trained with a neural network (NN), is used to reject the continuum events. The remaining background arises from:

- $B$ decays with two leptons in the final state, also this background is reduced with a $\mathrm{NN}$ technique;

- $B$ decays with a $J / \psi$ in the final state in which one of the leptons is misidentified, this background is suppressed with mass vetoes. 

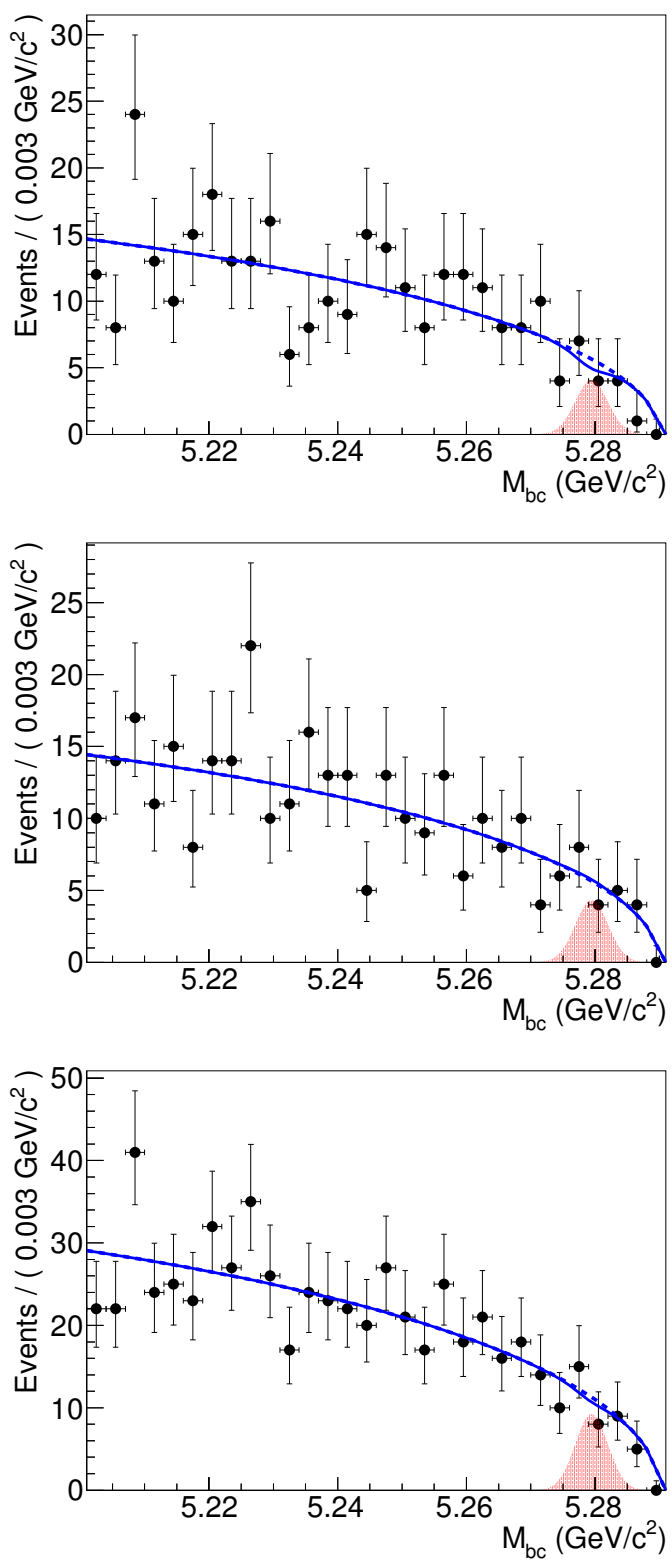

Figure 6: The $M_{b c}$ distributions for data events that pass the selection criteria for the decays $B^{0} \rightarrow K^{* 0} \mu^{+} e^{-}$(top), $B^{0} \rightarrow K^{* 0} \mu^{-} e^{+}$ (middle), and also both decays combined (bottom). Points with error bars are the data, and the blue solid curve is the result of the fit for the signal-plus-background hypothesis, where the blue dashed curve is the background component. The red shaded histogram represents the signal PDF with arbitrary normalization.

The signal yields are given by an unbinned extended maximum likelihood fit to the $M_{b c}$ distributions for the decays $B^{0} \rightarrow K^{* 0} \mu^{+} e^{-}, B^{0} \rightarrow K^{* 0} \mu^{-} e^{+}$, and also for both decays combined (see figure 6 ).

No excesses are observed and limits on the branching fractions at $90 \% \mathrm{CL}$ are given in Table 3. These results are the most stringent constraints on these LFV decays to date.
Table 3: Results from the fits. The columns correspond to the decay mode, efficiency, signal yield, $90 \%$ C.L. upper limit on the signal yield, and $90 \%$ C.L. upper limit on the branching fraction.

\begin{tabular}{l|cccc}
\hline Mode & $\begin{array}{c}\varepsilon \\
(\%)\end{array}$ & $N_{\text {sig }}$ & $N_{\text {sig }}^{\mathrm{UL}}$ & $\begin{array}{c}\mathcal{B}^{\mathrm{UL}} \\
\left(10^{-7}\right)\end{array}$ \\
\hline$B^{0} \rightarrow K^{* 0} \mu^{+} e^{-}$ & 8.8 & $-1.5_{-4.1}^{+4.7}$ & 5.2 & 1.2 \\
$B^{0} \rightarrow K^{* 0} \mu^{-} e^{+}$ & 9.3 & $0.4_{-4.5}^{+4.8}$ & 7.4 & 1.6 \\
$B^{0} \rightarrow K^{* 0} \mu^{ \pm} e^{\mp}$ (combined) & 9.0 & $-1.2_{-6.2}^{+6.8}$ & 8.0 & 1.8 \\
\hline
\end{tabular}

\section{Conclusions}

Although the observed deviations from LFU in $B$ meson decays are intriguing, their significance are still not sufficient at present to establish a violation unambiguously. However these hints, observed by three experiments operating in very different environments, have generated much interest within the theoretical community and several models of new physics which imply LFV, have been proposed.

The LHCb collaboration is working on updates of the measurements presented in these proceedings, using the full collected dataset. Concerning the LFU tests, other decay channels are being studied, these measure the ratios $\mathcal{R}(D), \mathcal{R}\left(D^{+}\right), \mathcal{R}\left(\Lambda_{c}^{+}\right), \mathcal{R}\left(D_{s}^{+}\right), \mathcal{R}(p p)$. They are analysed both in muonic and hadronic decay mode of the $\tau$ lepton. Concerning the LFV searches in $B$ decays, the LHCb collaboration is working on: $B^{0} \rightarrow K^{* 0} \tau \mu, B^{0} \rightarrow K^{* 0} e \mu, B_{s}^{0} \rightarrow$ $\phi \tau \mu, B_{s}^{0} \rightarrow \phi e \mu$, etc. Recently, the result about the search of the $B^{+} \rightarrow K^{+} e \mu$ decay has been published [36]

Finally, the large datasets that will be collected by the LHCb upgrade [16] and Belle II [14, 15] experiments will allow tests of LFU in FCNC and in tree level semitauonic $B$ transitions precise enough to confirm or reject LFU breaking.

\section{References}

[1] S.L. Glashow, D. Guadagnoli, K. Lane, PRL 114, 091801 (2015)

[2] G. Hiller, D. Loose, K. Schonwald, JHEP 12, 027 (2016)

[3] BaBar Collaboration, NIM A479, 117-232 (2002)

[4] Belle Collaboration, NIM A479, 1-116 (2002)

[5] LHCb Collaboration, JINST 3, 508005 (2008)

[6] M. Bordone, G. Isidori, A. Pattori, EPJ C76, 440 (2016)

[7] Belle Collaboration, PRL 103, 171801 (2009)

[8] BaBar Collaboration, PRD 86, 032012 (2012)

[9] LHCb Collaboration, JHEP 05, 159 (2013)

[10] LHCb Collaboration, PRL 113,151601 (2014)

[11] LHCb Collaboration, JHEP 08, 055 (2017)

[12] LHCb Collaboration, PRL 122, 191801 (2019)

[13] Belle Collaboration, arXiv:1904.02440v2

[14] Belle II Collaboration, arXiv:1011.0352

[15] Belle II Collaboration, arXiv:1808.10567

[16] LHCb Collaboration, LHCb-TDR 13,14,15,66

[17] BaBar Collaboration, PRL 109, 101802 (2012) 
[18] Belle Collaboration, PRD 92, 072014 (2015)

[19] LHCb Collaboration, PRL 115, 111803 (2015)

[20] Belle Collaboration, PRD 94, 072007 (2016)

[21] Belle Collaboration, PRL 118, 211801 (2017)

[22] LHCb Collaboration, PRL 97, 072014 (2018)

[23] LHCb Collaboration, PRL 120, 171802 (2018)

[24] LHCb Collaboration, PRL 120, 121801 (2018)

[25] Belle Collaboration, arXiv:1910.05864

[26] https://hflav-eos.web.cern.ch/hflaveos/semi/spring19/html/RDsDsstar/RDRDs.html

[27] L. Calibbi, G. Signorelli, Riv. Nuovo Cimento 41, 1 (2018)

[28] A. Crivellin, L. Hofer, J. Matias, U. Nierste, S. Pokorski, J. Rosiek, PR D 92, 054013 (2015)
[29] B.Beveric, S. Fajfer, N. Kosnik, O. Sumensari, PR D 94, 115021 (2016)

[30] I. de Medeiros Varzielas, G. Hiller, JHEP 06, 072 (2015)

[31] LHCb Collaboration, PRL 111, 141801 (2013)

[32] LHCb Collaboration, JHEP 03, 078 (2018)

[33] LHCb Collaboration, PRL 123, 211801 (2019)

[34] BaBar Collaboration, PRD 77, 091104 (2008)

[35] ALEPH Collaboration,Phys. Rept. 421, 191 (2005)

[36] LHCb Collaboration, PRL 123, 241802 (2019)

[37] BaBar Collaboration, PRD 73, 092001 (2006)

[38] Belle Collaboration, PRD 98, 071101 (2018) 